\title{
Infection: musculoskeletal
}

\author{
Diego Jaramillo
}

Received: 30 December 2010 / Accepted: 4 January 2011

(C) Springer-Verlag 2011

\begin{abstract}
The imaging approach to osteomyelitis has evolved in the past two decades. Advances in MRI allow for whole body imaging, decreasing the need for scintigraphy when symptoms are not localized or the disease may be multifocal. There is an increasing clinical need for depiction of abscesses in the soft tissues and subperiosteal space, particularly because methicillin-resistant Staphylococcus aureus infections constitute more than one-third of all the infections. The increasing emphasis on radiation dose reduction has also led away from scintigraphy and computed tomography. MR imaging has become the advanced imaging modality of choice in osteomyelitis. There is an increasing understanding of the appropriate role for gadolinium enhancement, which is not indicated when the pre-gadolinium images are normal. Other related infections, including pyomyositis, are best imaged with MRI.
\end{abstract}

Keywords Infection $\cdot$ Musculoskeletal $\cdot$ Children $\cdot$ MRI

\section{Epidemiology}

Acute hematogenous osteomyelitis is the most common form of musculoskeletal infection [1]. Its incidence is difficult to determine, but it has been reported to be 1 per 5,000 [2]. There is conflicting data regarding the change of the disease over time. A study in 1998 found that the

Disclaimer The supplement this article is part of is not sponsored by the industry. Dr. Jaramillo has no financial interests, investigational or off-label uses to disclose.

\section{Jaramillo $(\bowtie)$}

Department of Radiology, Children's Hospital of Philadelphia, 34th Street and Civic Center Boulevard, 3NW17, Philadelphia, PA 19104, USA

e-mail: jaramillo@email.chop.edu incidence in Norway ranged between 70 and 160 per million and that the incidence had remained stable between 1965 and 1994 [3]. An American study, however, found that in the last 20 years the incidence of osteomyelitis had increased 2.8-fold, whereas that of septic arthritis was stable. Methicillin-resistant Staphylococcus aureus (MRSA) was the causative organism in $30 \%$ of the children [4]. More than half of the cases occur before 5 years of age, and it appears to be more frequent in boys. Approximately $70 \%$ of infections involve the lower extremities and onethird affect the knee [2].

\section{Pathophysiology}

Hematogenous osteomyelitis results from the seeding of bacteria, usually Staphylococcus aureus (S. aureus) in the metaphysis of a long bone (Fig. 1) or in the metaphyseal equivalents of the axial skeleton (Fig. 2). S. aureus bacteria, which are present in the skin or mucosal membranes, enter the bloodstream and lodge in the bone, usually at sites where blood flow is abundant and slow. The metaphyses are richly vascularized and the valveless sinusoidal loops of the metaphyseal venules have sluggish flow and discontinuous endothelium which facilitates bacterial invasion. During childhood, the metaphyses that grow the fastest, such as those around the knee, are the most richly vascularized in order to produce new bone at a rapid rate. These rapidly growing metaphyses also tend to be affected more frequently by infection. $S$. aureus has the capacity to attach to the bony matrix and produce proteolytic enzymes that destroy the surrounding tissues, allowing the spread of the infection. From the bone, the infection extends through the porous metaphyseal cortex into the subperiosteal space. The periosteum in children is loosely attached to the bone and separates easily from it, creating a space that allows the accumulation 
Fig. 1 An 11-year-old boy with 1-week history of ankle pain, erythema and swelling. a Sagittal fat-suppressed gadoliniumenhanced T1-W image shows that there is diffuse marrow enhancement in the metaphysis and epiphysis of the distal tibia, with two central foci of decreased signal intensity (arrow) indicating early abscess formation. b Axial fat-suppressed gadolinium-enhanced T1-W image shows that the metaphyseal abscess is extending toward the cortex (arrow), almost becoming a draining sinus
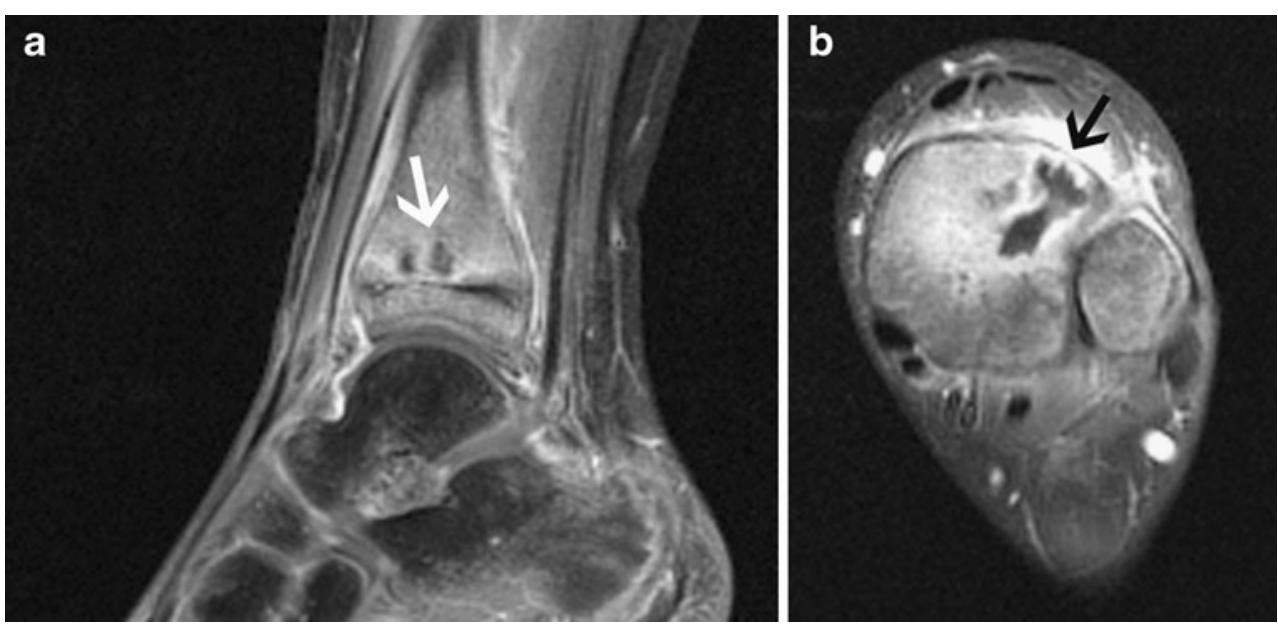

of purulent collections (Figs. 3, 4 and 5). A rapid elevation of the periosteum can lead to devascularization of the bone, which explains why in some cases of infection the bone scan is cold and the post-gadolinium image shows absence of enhancement.

In the flat bones, at the junction of cartilage and bone, there are "metaphyseal equivalents," areas with endochondral ossification that are richly vascularized and thus also prone to infection [5]. A metaphyseal type of vascularity is also seen in the periphery of the round bones and of the secondary ossification centers.

In infants before 18 months of age, there are transphyseal vessels that allow the spread of infection from the metaphysis to the epiphysis [6]. This explains why infantile osteomyelitis frequently results in the destruction of the epiphysis and damage to the adjacent joint. Direct spread of infection from the epiphysis, however, is not the main cause of septic arthritis. More frequently there appears to be hematogenous seeding of the synovial vascularity in the periphery of the joint.

A metaphyseal hematoma due to trauma appears to predispose to bacterial colonization and a history of trauma is reported in one-third of cases of acute hematogenous osteomyelitis. There are other host-related factors that predispose a patient to bone infections including premature birth, umbilical catheterization, urinary tract infection, immunodeficiency and other pre-existing diseases [7].

Specific features of the invading bacteria can also determine the clinical and radiological picture. The presence of genes encoding Panton-Valentine leukocidin (pvl) [8], seen primarily in MRSA infections, is associated with higher erythrocyte sedimentation rate (ESR), higher $\mathrm{C}$ reactive protein (CRP) concentration and a higher frequency of positive blood cultures. Infections with pvl bacteria also have a higher incidence of associated subperiosteal collections, myositis or pyomyositis (Fig. 4) [8].

\section{Clinical considerations}

A definitive diagnosis of osteomyelitis requires the recovery of the organism from the focus of metaphyseal or subperiosteal infection, or a positive blood culture in a child with a clinical and radiographical picture consistent with clinical infection [9].

Established osteomyelitis is associated with leukocytosis in the range of $12-15,000 / \mathrm{mcL}$, and an ESR in the range of $30-50 \mathrm{~mm} / \mathrm{hr}$ [10]. There is an increasing reliance placed on the CRP level, as its elevation precedes that of the ESR, usually within a day of the beginning of the infection [10] and it also declines sooner in response to therapy. CRP also is a valuable indicator of complications. In a recent series, subperiosteal abscesses only occurred in children with a CRP greater than $3.6 \mathrm{mg} / \mathrm{dL}$. Children with MRSA infection and a CRP greater than $6 \mathrm{mg} / \mathrm{dL}$ have a $40 \%$ chance of having deep venous thrombosis [11, 12].

In neonates osteomyelitis can be caused by E. Coli, Enterobacteriae, Group B Streptococci, and Candida Albicans; is more often multifocal; extends frequently into the epiphysis, and can result in areas of decreased perfusion manifested as cold scintigrams. In young children, milder osteomyelitis can be due to Kingella Kingae [13].

\section{Diagnostic modalities}

A comparison of the relative sensitivities and specificities of the various modalities would be useful, but a valid evaluation would need to be prospective, with all imaging modalities performed almost simultaneously in a large number of patients. To the best of my knowledge, such a study has not been carried out, and in most comparisons either scintigraphy was done first [14] or MRI was used only in complicated cases [15]. 

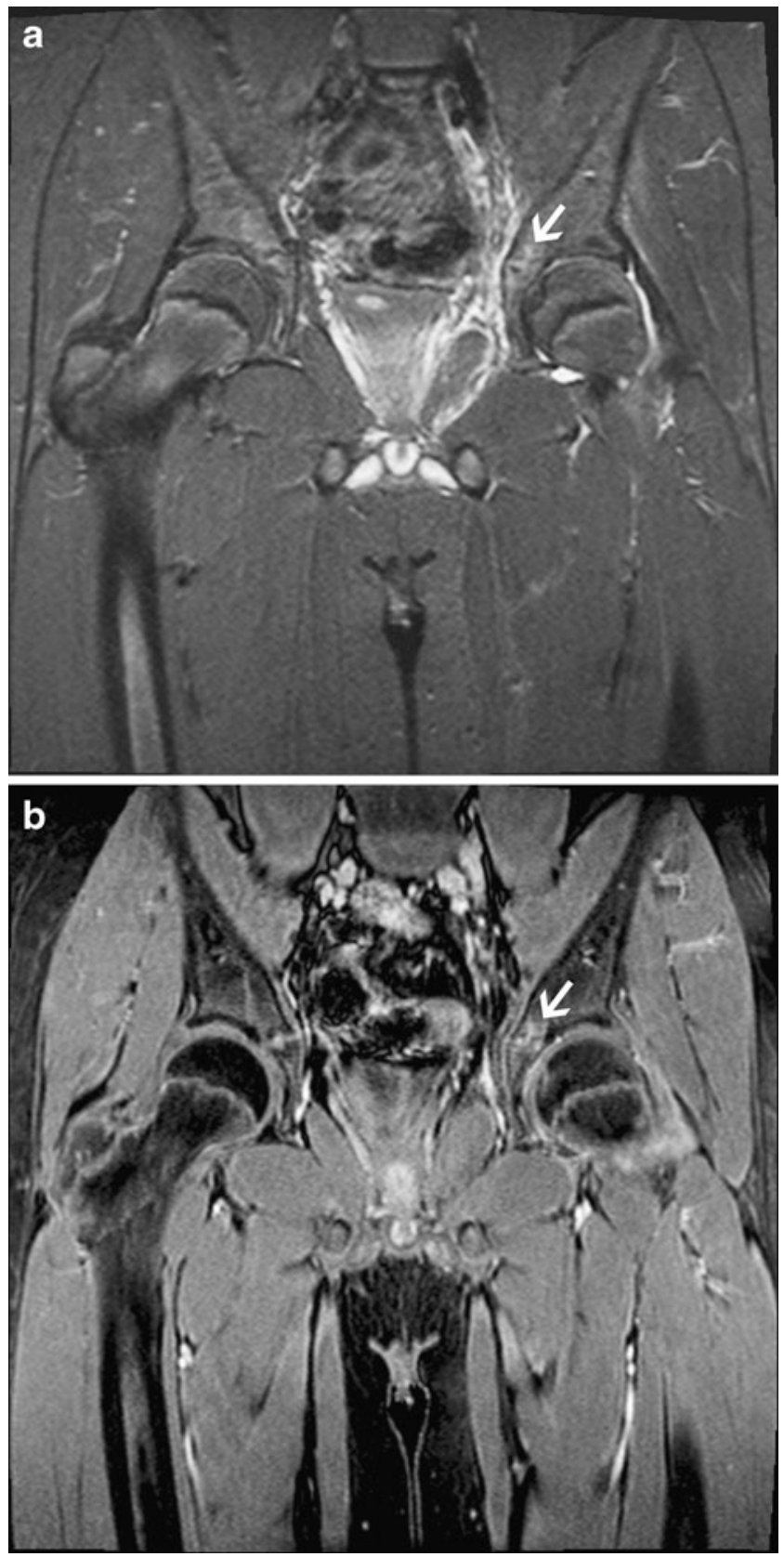

Fig. 2 A 12-year-old girl with pain in the left hip and fever. a Coronal STIR image shows that there is a subtle focus of high signal intensity primarily on the iliac side of the triradiate cartilage (arrow). There is adjacent soft tissue swelling of the pelvic soft tissues and a small left joint effusion. b Coronal gradient recalled echo fat-suppressed postgadolinium T1-W image shows that the area of abnormality is located in the metaphyseal equivalent (arrow) of the left iliac wing

Plain radiography is insensitive for the evaluation of osteomyelitis, but necessary to exclude other pathologies such as injuries or tumors [16]. When positive, however, plain radiographs can be extremely helpful in the subsequent evaluation. Destruction of the bone, typically manifested as focal metaphyseal radiolucency, becomes apparent $7-10$ days after the beginning of symptoms.
Unfortunately, only $20 \%$ of cases have abnormal radiographical findings by the second week [17]. Earlier, deep soft tissue swelling may be visible, but this is difficult to detect. There is visible elevation of the periosteum, which also appears late.

MR imaging has become the predominant modality for the evaluation of bone infections. In osteomyelitis, the first question related to imaging is whether there is bone involvement and, if so, whether there is an abscess that requires drainage. The earliest manifestation of bone infection on MR images is edema, which radiates from the initial focus of disease into the adjacent marrow and soft tissues. T2-weighted and short tau inversion recovery (STIR) images are highly sensitive for the diagnosis of early osteomyelitis, seen as high signal intensity in the metaphysis (Fig. 3). In the pelvis, the abnormal areas are located in metaphyseal equivalents and are small relative to the extent of soft tissue involvement [18].

The second question is whether there is an abscess. A purulent collection will present on gadolinium-enhanced images as a low signal intensity nonenhancing center surrounded by a halo of enhancing tissue. Sometimes there may be small pus collections. Smaller collections less than a few centimeters in size respond to antibiotics (Fig. 1), but larger purulent collections need to be drained. The decision to intervene is based on the clinical response as much as on the volume of the collection.

Osteomyelitis should be differentiated from normal hematopoietic marrow, which is also metaphyseal and contains abundant water. Normal hematopoietic marrow should be similar in all metaphyses imaged and should be of higher signal intensity than muscle on T1-weighted images. Extensive soft tissue involvement is a good differentiating point between osteomyelitis and stress injury, in which the edema is more confined to the bone. Osteomyelitis should also be differentiated from tumor. A soft tissue mass, a sharp transition between normal and abnormal marrow and invasion of adjacent structures suggest a tumor, whereas an abscess, a draining sinus and extensive perilesional edema suggest infection [19]. A good differentiating feature between acute osteomyelitis and other marrow-invading processes is the presence of fat globules within the lesion (Fig. 3c). On T1-weighted images, small rounded areas of high signal intensity can be seen within the bone, subperiosteal space and soft tissue as a frequent finding in acute osteomyelitis. It is likely that increased intramedullary pressure leads to necrosis of the adipose tissue and release of free fatty globules. Although not pathognomonic, fatty globules on MR imaging support the diagnosis of osteomyelitis over other marrow disorders such as neoplasia [20].

There is controversy regarding when to use gadolinium enhancement in infants and children with suspected 

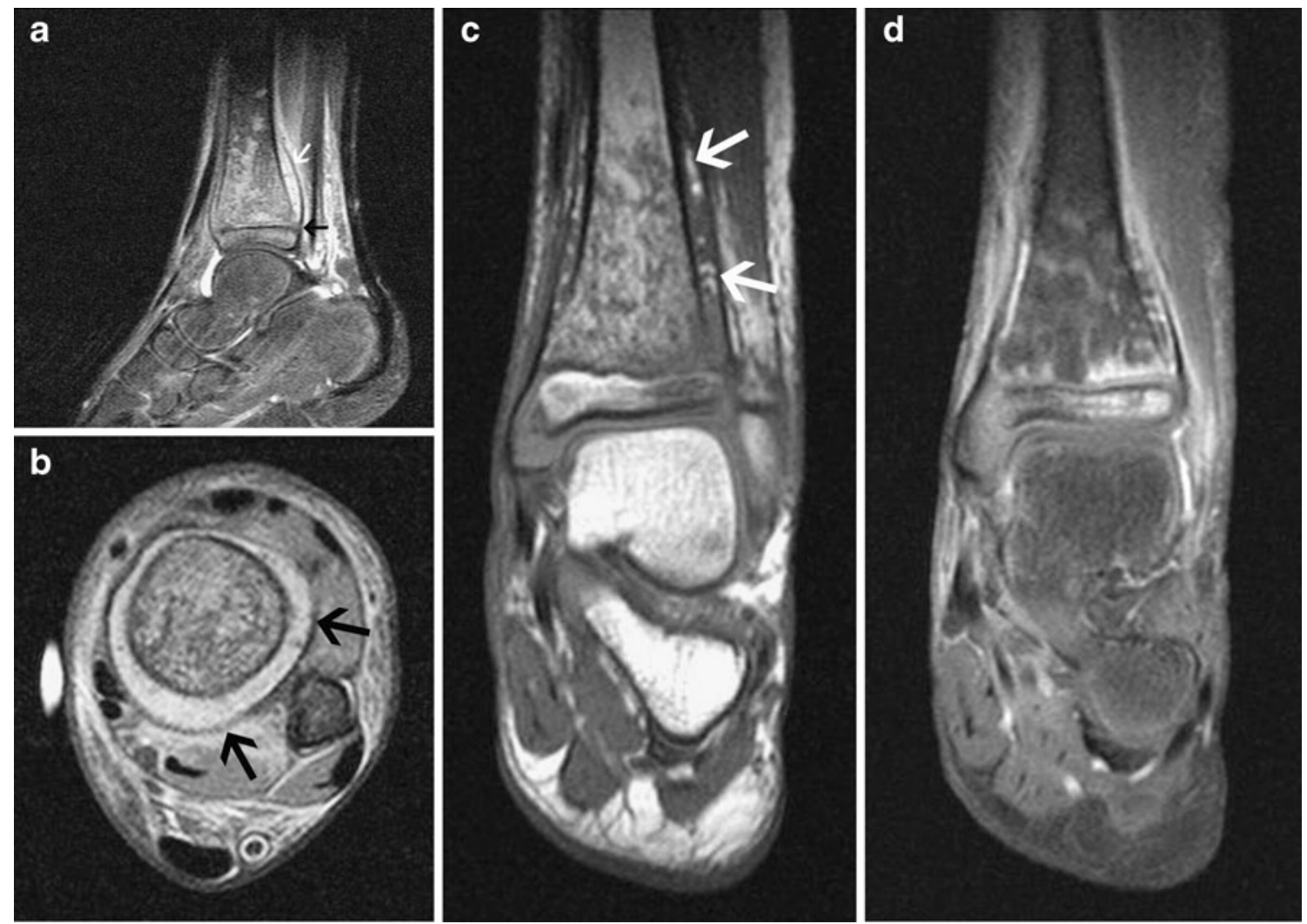

Fig. 3 A 10-year-old girl with persistent fever after 3 days of antibiotic therapy. a Sagittal STIR image shows increased signal intensity in the distal tibial marrow primarily involving the metaphysis. There is associated edema of the surrounding tissues and an ankle effusion. The periosteum (white arrow) is separated from the bone by a subperiosteal fluid collection. The elevated periosteum joins the bone in the perichondral region (black arrow). b Axial T2-W

osteomyelitis. Gadolinium enhancement does not increase the sensitivity or specificity for diagnosis of osteomyelitis. If the water-sensitive images are normal, gadolinium enhancement is of no value [21,22]. One exception is an abscess involving the epiphyseal cartilage alone, in which unenhanced images can be negative [23]. If the watersensitive images are abnormal, however, gadolinium enhancement is of value in increasing confidence in the diagnosis of an abscess [21] and allowing the planning of the abscess aspiration and drainage.

On sonography, the earliest manifestation of osteomyelitis is juxtacortical soft tissue swelling with early periosteal thickening. Early inflammatory changes are depicted as areas of hyperemia on power Doppler or color Doppler imaging $[24,25]$. Sonography is particularly valuable for the diagnosis of early subperiosteal and soft tissue abscesses (Fig. 4). The elevated periosteum is seen as an echogenic line separated from the echogenic cortex by a purulent collection of mixed echogenicity [26]. If there is any doubt regarding whether an echogenic interface is the periosteum image shows a halo of fluid surrounding the cortex and limited by the periosteum (arrows). c Coronal T1-W image shows round areas of high signal intensity (arrows) representing fat globules, a finding highly suggestive of infection. d Coronal fat-suppressed postgadolinium T1-W image shows decreased enhancement in the marrow space of the distal tibia, a common finding in osteomyelitis

[27], it can be followed to the region of the physis and invariably it will come to merge with the physis at the site of the tight perichondral attachments. In later cases, sonography can show cortical destruction. Unfortunately, however, ultrasound provides limited depiction of intraosseous pathology and is thus not the modality of choice.

Scintigraphy using ${ }^{99 \mathrm{~m}} \mathrm{Tc}$-methylene diphosphonate has a sensitivity of $80 \%$ and a specificity of only $50 \%$ [28]. The sensitivity has decreased as infections that have marked soft tissue involvement have increased. Currently, the sensitivity may be as low as 53\% [29]. FDG PET appears to be considerably more sensitive (95\%) and specific (87\%). Unfortunately, PET has limited availability. Given that both techniques deliver radiation and that the capability of whole-body imaging is now also available with MR imaging, MR imaging is a superior technique in children $[30,31]$. Other scintigraphic modalities, such as white blood cells labeled with indium 111 or ${ }^{99 \mathrm{~m}} \mathrm{Tc}$ hexamethylpropyleneamine oxime, require relatively large volumes of blood and are not used frequently in younger children [28]. 
Fig. 4 A 13-month-old boy with history of fever and thigh pain. The patient has sickle cell disease. a Sagittal section through the mid-thigh shows that the periosteum (top arrow) is separated from the cortex (bottom arrow) by a sonolucent subperiosteal fluid collection consistent with a subperiosteal abscess. b Axial images through the left thigh show marked edema of the deep quadriceps compared to the right. An echogenic line (arrow) corresponds to an elevated periosteum. c Axial fat-suppressed T2-W image shows the periosteum (arrow) is elevated by the subperiosteal abscess and that there are extensive inflammatory changes surrounding the abnormal marrow
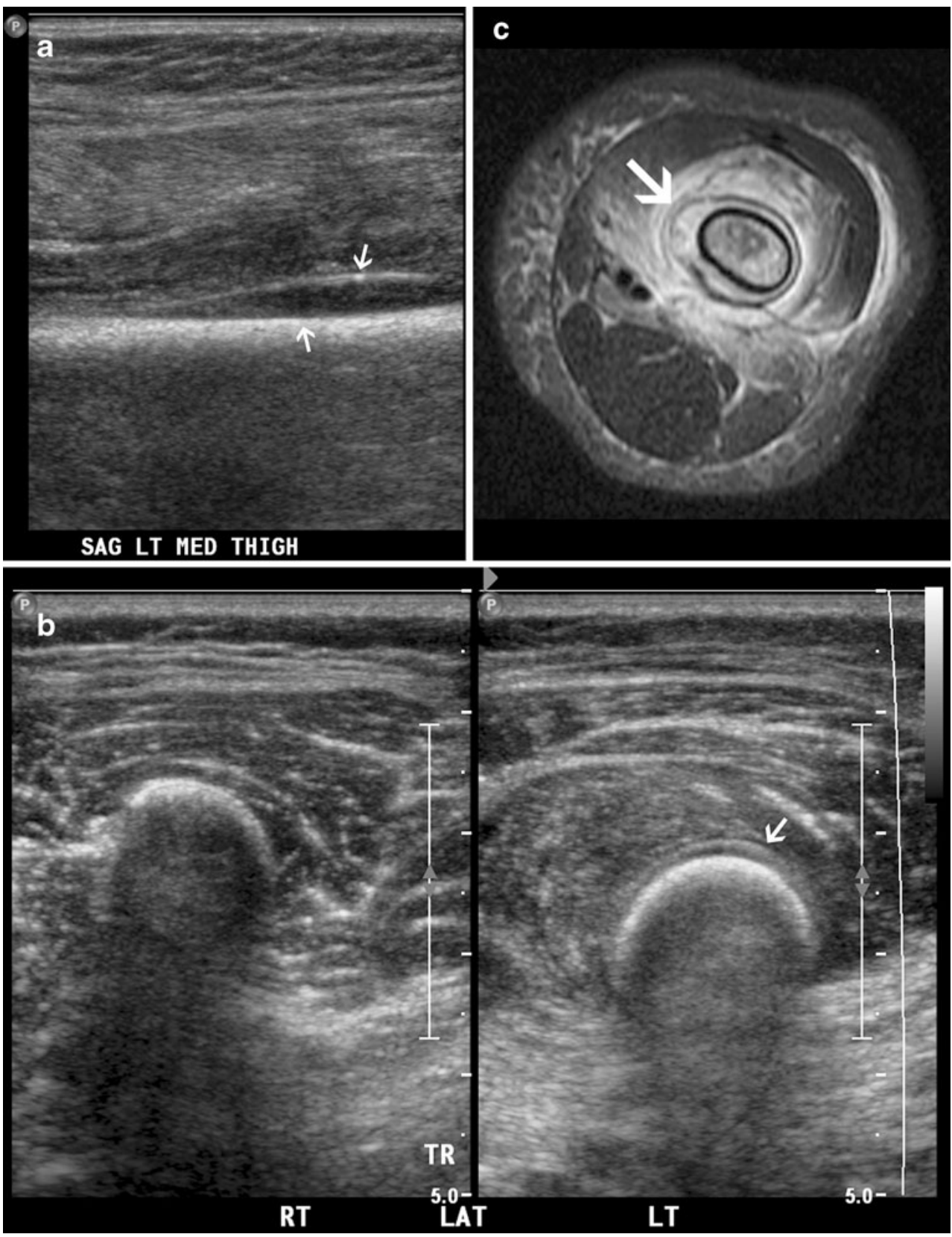

CT is not a primary modality for imaging osteomyelitis. On occasion, children with pelvic osteomyelitis present with pelvic pain and it is important to search for evidence of inflammatory changes in the pelvic wall musculature to exclude pelvic osteomyelitis [18]. In chronic osteomyelitis, CT can help in the evaluation of a sequestrum, which can be difficult to locate on MR imaging.

\section{Determination of the extent of disease}

In recent years, osteomyelitis has become more frequently associated with subperiosteal abscess, pyomyo- sitis, deep venous thrombosis and septic arthritis with more patients requiring surgical intervention. A recent series found that nearly $70 \%$ of community-acquired (CA) S. aureus infections [29] were associated with such extraosseous findings. An important contributor has been the rise of community-acquired MRSA, which now accounts for $30 \%-40 \%$ of osteomyelitis cases [32]. In patients with CA MRSA, two series reported rates of surgery greater than $90 \%[29,32]$. Another ominous trend has been the increasing involvement of the cartilaginous epiphyses in younger patients [23]. The greater need to intervene and the greater frequency of extraosseous involvement suggest that imaging of osteomyelitis should 


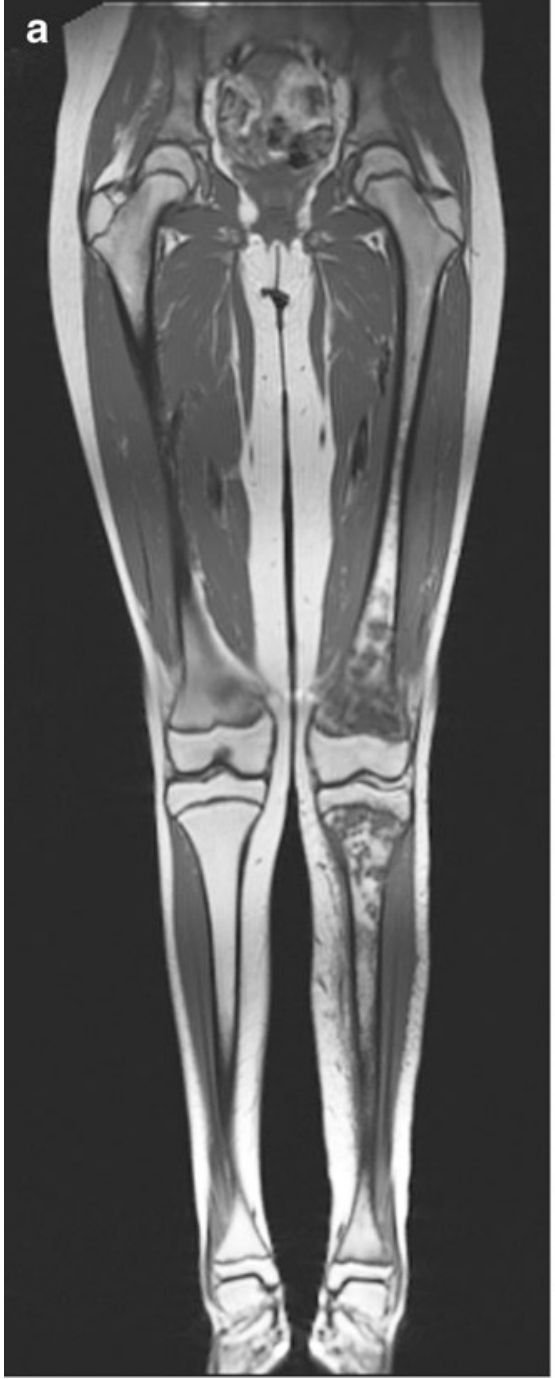

b

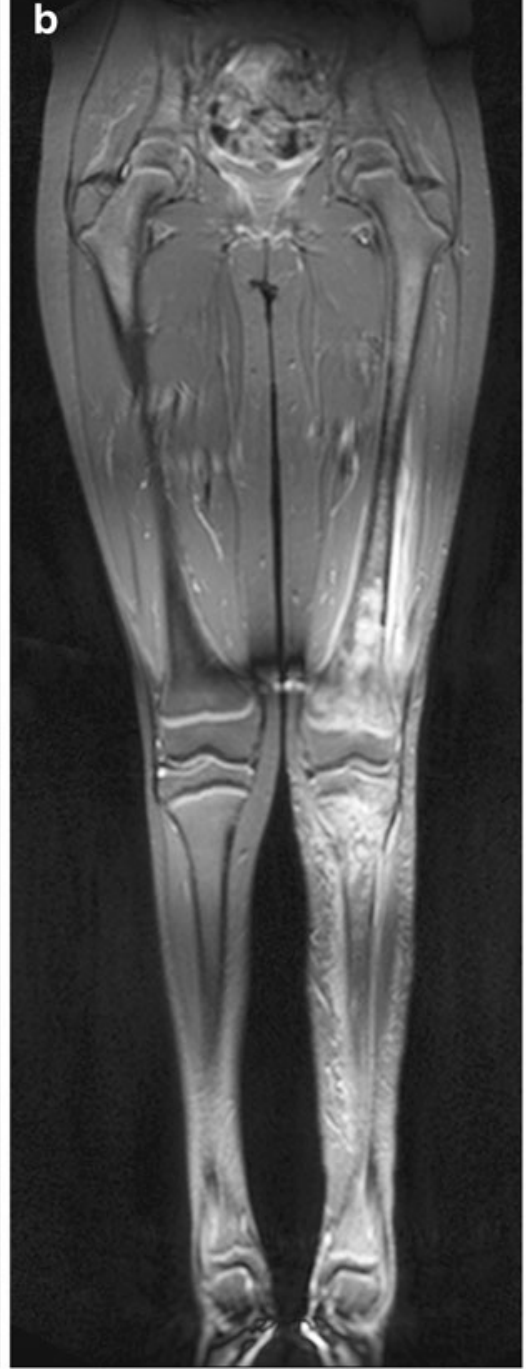

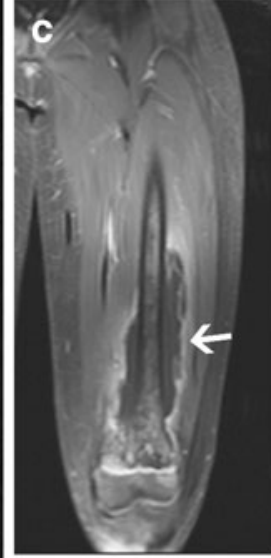

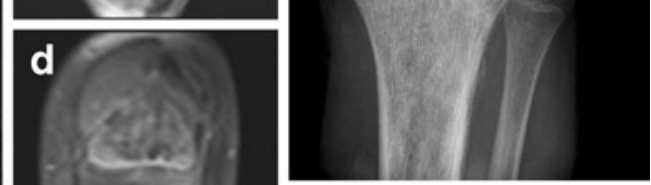

Fig. 5 A 12-year-old girl, previously healthy, with fever and left knee swelling. The patient has nodules on chest $\mathrm{CT}$ and MRSA bacteremia. a Coronal $\mathrm{T} 1 \mathrm{-W}$ image of the lower extremities shows marrow abnormality in the left lower extremity, primarily in the distal left femur and proximal tibia. b Coronal STIR confirms the marrow edema and surrounding soft tissue swelling. c Coronal fat-suppressed post-gadolinium T1-W image of the left femur shows a very large subperiosteal nonenhancing fluid collection (arrow). d Coronal fatsuppressed post-gadolinium T1-W image of the left tibia shows a very large subperiosteal nonenhancing fluid collection (arrow). e Frontal radiograph shows sclerosis and abnormal bony texture in the distal femur and proximal tibia. This radiograph was obtained slightly more than 4 weeks after the first MRI. Initial radiographs were normal not be confined to the bone but should also evaluate the cartilaginous structures, surrounding soft tissues and adjacent vessels.

There are specific imaging considerations when osteomyelitis affects certain anatomical locations. Spinal osteomyelitis always requires the use of gadolinium-enhanced MR imaging. Contrast-enhanced MRI is essential in the differentiation of abscess in the epidural space or paravertebral masses from inflammatory masses. A follow-up gadolinium-enhanced MR study is useful in cases when the initial study is equivocal and the symptoms persist [33].
In pelvic osteomyelitis, the initial focus of bone infection is usually subtle, and in almost every case it occurs in a metaphyseal equivalent [18] (Fig. 2). Five of these regions, adjacent to the sacroiliac joint, triradiate cartilage, pubic symphysis, ischiopubic synchondrosis and iliac apophyses, are most commonly affected [34]. MR imaging is particularly useful in this condition because the geometry is complex, the primary focus of disease is difficult to detect and the incidence of associated soft tissue abnormalities is high [14]. In patients with acute hip pain, no evidence of septic arthritis and a clinical picture suggestive of infection, 
performing a pelvic MRI often yields the diagnosis of osteomyelitis or pyomyositis [35].

\section{Imaging of complications}

In children, most uncomplicated osteomyelitis responds to antibiotic therapy and less than $5 \%$ of patients develop chronic infections. A delay greater than 4 days in the initiation of antibiotic therapy increases the likelihood of sequelae, such as deformity and decreased function [36]. Chronic osteomyelitis may present with a relatively small focus of disease surrounded by a strong reaction of the host bone, which walls off the infection. This is termed a Brodie abscess, and is characterized by a necrotic center, a well-defined inner border, and a hazy, ill-defined and broader halo of infection [37]. A less well-contained focus of osteomyelitis can develop a necrotic center or sequestrum, surrounded by pus. In turn, it is walled off by reactive bone or involucrum. The third type of osteomyelitis is a sclerotic form in which the bone is uniformly dense. Chronic infection is most commonly due to $S$. aureus. In a large series, the tibia was the bone more commonly affected [38]. It is difficult to determine by imaging whether chronic infection is active. Persistent high signal intensity on water-sensitive images or persistent enhancement suggests infection. PET has high specificity for active infection [28] and may be of help in this setting.

Chronic bacterial osteomyelitis should be differentiated from chronic recurrent multifocal osteomyelitis (CRMO), a nonbacterial inflammatory disease involving multiple osseous structures, typically the metaphysis of long bones and the clavicle [39]. Whole-body MRI is a useful modality for the evaluation of this disease; symmetric involvement is highly suggestive of this condition [40], which responds to antiinflammatory agents rather than antibiotics. Chronic osteomyelitis due to $S$. aureus should also be differentiated from disease caused by other organisms such as tuberculosis and Bartonella hensealae (cat-scratch disease) [41].

\section{Role of imaging in guiding therapy}

During the last few years, several developments have changed the imaging approach to osteomyelitis. First, whole-body MRI can now detect multifocal disease or abnormalities that are not well-localized [30, 31]. There is less of an advantage in using scintigraphy for those cases in which there may be disseminated disease or in which there are no focal symptoms. Second, growing concern about exposing children to radiation has led to increasing substitution of MRI and sonography for scintigraphy and
CT. Third, MRSA has changed the presentation of the disease, with a significant percentage of patients having soft tissue findings and decreased vascularization, both of which are better depicted by MRI [29]. Finally, MRI has become more readily available, even on an emergency basis. In summary, there are increasing reasons to use MRI as the second modality following radiographs, using whole-body MRI sequences when there is suspicion of multifocal disease or in infants when it is difficult to ascertain the location of the disease.

Once the diagnosis of osteomyelitis is established, serial MR imaging studies appear to be of limited value in terms of affecting management [11]. On patients who do not respond to therapy or who have a persistently elevated CRP level, a repeat MRI may help by showing the development of an abscess.

\section{Conclusion}

The evaluation of osteomyelitis continues to be challenging. Changes related to the increased prevalence of MRSA, advances in imaging and changes in therapeutic approaches have modified the diagnostic approach to this disease.

\section{References}

1. Grimprel E, Cohen R (2007) Epidemiology and physiopathology of osteoarticular infections in children (newborns except). Arch Pediatr 14(Suppl 2):S81-S85

2. Steer AC, Carapetis JR (2004) Acute hematogenous osteomyelitis in children: recognition and management. Paediatr Drugs 6:333-346

3. Dahl LB, Hoyland AL, Dramsdahl H et al (1998) Acute osteomyelitis in children: a population-based retrospective study 1965 to 1994 . Scand J Infect Dis 30:573-577

4. Gafur OA, Copley LA, Hollmig ST et al (2008) The impact of the current epidemiology of pediatric musculoskeletal infection on evaluation and treatment guidelines. J Pediatr Orthop 28:777-785

5. Nixon GW (1978) Hematogenous osteomyelitis of metaphysealequivalent locations. AJR 130:123-129

6. Ogden JA, Lister G (1975) The pathology of neonatal osteomyelitis. Pediatrics 55:474-478

7. Ranson M (2009) Imaging of pediatric musculoskeletal infection. Semin Musculoskelet Radiol 13:277-299

8. Bocchini CE, Hulten KG, Mason EO Jr et al (2006) PantonValentine leukocidin genes are associated with enhanced inflammatory response and local disease in acute hematogenous Staphylococcus aureus osteomyelitis in children. Pediatrics 117:433-440

9. Lew DP, Waldvogel FA (2004) Osteomyelitis. Lancet 364:369379

10. Conrad DA (2010) Acute hematogenous osteomyelitis. Pediatr Rev 31:464-471

11. Courtney PM, Flynn JM, Jaramillo D et al (2010) Clinical indications for repeat MRI in children with acute hematogenous osteomyelitis. J Pediatr Orthop 30:883-887 
12. Hollmig ST, Copley LA, Browne RH et al (2007) Deep venous thrombosis associated with osteomyelitis in children. J Bone Joint Surg Am 89:1517-1523

13. Offiah AC (2006) Acute osteomyelitis, septic arthritis and discitis: differences between neonates and older children. Eur J Radiol 60:221-232

14. Connolly LP, Connolly SA, Drubach LA et al (2002) Acute hematogenous osteomyelitis of children: assessment of skeletal scintigraphy-based diagnosis in the era of MRI. J Nucl Med 43:1310-1316

15. Malcius D, Jonkus M, Kuprionis G et al (2009) The accuracy of different imaging techniques in diagnosis of acute hematogenous osteomyelitis. Medicina (Kaunas) 45:624-631

16. Jaramillo D, Treves ST, Kasser JR et al (1995) Osteomyelitis and septic arthritis in children: appropriate use of imaging to guide treatment. AJR 165:399-403

17. Capitanio MA, Kirkpatrick JA (1970) Early roentgen observations in acute osteomyelitis. Am J Roentgenol Radium Ther Nucl Med 108:488-496

18. Connolly SA, Connolly LP, Drubach LA et al (2007) MRI for detection of abscess in acute osteomyelitis of the pelvis in children. AJR 189:867-872

19. Jaramillo D, Laor T (2008) Pediatric musculoskeletal MRI: basic principles to optimize success. Pediatr Radiol 38:379-391

20. Davies AM, Hughes DE, Grimer RJ (2005) Intramedullary and extramedullary fat globules on magnetic resonance imaging as a diagnostic sign for osteomyelitis. Eur Radiol 15:2194-2199

21. Averill LW, Hernandez A, Gonzalez L et al (2009) Diagnosis of osteomyelitis in children: utility of fat-suppressed contrastenhanced MRI. AJR 192:1232-1238

22. Kan JH, Young RS, Yu C et al (2010) Clinical impact of gadolinium in the MRI diagnosis of musculoskeletal infection in children. Pediatr Radiol 40:1197-1205

23. Johnson DP, Hernanz-Schulman M, Martus JE et al (2010) Significance of epiphyseal cartilage enhancement defects in pediatric osteomyelitis identified by MRI with surgical correlation. Pediatr Radiol DOI. doi:10.1007/s00247-010-1849-6, Oct 9 Epub ahead of print

24. Collado P, Naredo E, Calvo C et al (2008) Role of power Doppler sonography in early diagnosis of osteomyelitis in children. J Clin Ultrasound 36:251-253

25. Keller MS (2005) Musculoskeletal sonography in the neonate and infant. Pediatr Radiol 35:1167-1173, quiz 1293

26. Chau CL, Griffith JF (2005) Musculoskeletal infections: ultrasound appearances. Clin Radiol 60:149-159

27. Kaiser S, Rosenborg M (1994) Early detection of subperiosteal abscesses by ultrasonography. A means for further successful treatment in pediatric osteomyelitis. Pediatr Radiol $24: 336-339$

28. Gotthardt M, Bleeker-Rovers CP, Boerman OC et al (2010) Imaging of inflammation by PET, conventional scintigraphy, and other imaging techniques. J Nucl Med 51:1937-1949

29. Browne LP, Mason EO, Kaplan SL et al (2008) Optimal imaging strategy for community-acquired Staphylococcus aureus musculoskeletal infections in children. Pediatr Radiol 38:841-847

30. Jaramillo D (2010) Whole-body MR imaging, bone diffusion imaging: how and why? Pediatr Radiol 40:978-984

31. Darge K, Jaramillo D, Siegel MJ (2008) Whole-body MRI in children: current status and future applications. Eur J Radiol 68:289-298

32. Arnold SR, Elias D, Buckingham SC et al (2006) Changing patterns of acute hematogenous osteomyelitis and septic arthritis: emergence of community-associated methicillin-resistant Staphylococcus aureus. J Pediatr Orthop 26:703-708

33. Dunbar JA, Sandoe JA, Rao AS et al (2010) The MRI appearances of early vertebral osteomyelitis and discitis. Clin Radiol 65:974-981

34. McPhee E, Eskander JP, Eskander MS et al (2007) Imaging in pelvic osteomyelitis: support for early magnetic resonance imaging. J Pediatr Orthop 27:903-909

35. Karmazyn B, Loder RT, Kleiman MB et al (2007) The role of pelvic magnetic resonance in evaluating nonhip sources of infection in children with acute nontraumatic hip pain. J Pediatr Orthop 27:158-164

36. McCarthy JJ, Dormans JP, Kozin SH et al (2005) Musculoskeletal infections in children: basic treatment principles and recent advancements. Instr Course Lect 54:515-528

37. Jones HW, Harrison JW, Bates J et al (2009) Radiologic classification of chronic hematogenous osteomyelitis in children. J Pediatr Orthop 29:822-827

38. Beckles VL, Jones HW, Harrison WJ (2010) Chronic haematogenous osteomyelitis in children: a retrospective review of 167 patients in Malawi. J Bone Joint Surg Br 92:1138-1143

39. Girschick HJ, Zimmer C, Klaus G et al (2007) Chronic recurrent multifocal osteomyelitis: what is it and how should it be treated? Nat Clin Pract Rheumatol 3:733-738

40. Fritz J, Tzaribatchev N, Claussen CD et al (2009) Chronic recurrent multifocal osteomyelitis: comparison of whole-body MR imaging with radiography and correlation with clinical and laboratory data. Radiology 252:842-851

41. de Kort JG, Robben SG, Schrander JJ et al (2006) Multifocal osteomyelitis in a child: a rare manifestation of cat-scratch disease: a case report and systematic review of the literature. J Pediatr Orthop B 15:285-288 